\title{
The use of Multiple Breath Washout for Assessing Cystic Fibrosis in Infants
}

\section{Dr Gwyneth Davies \& Dr Paul Aurora}

To cite this article: Dr Gwyneth Davies \& Dr Paul Aurora (2016): The use of Multiple Breath Washout for Assessing Cystic Fibrosis in Infants, Expert Review of Respiratory Medicine

To link to this article: http://dx.doi.org/10.1080/17476348.2017.1269604

Accepted author version posted online: 07

Dec 2016.

Submit your article to this journal $\pi$

Џll Article views: 2

Q View related articles $\longleftarrow$

View Crossmark data ¿ 
Publisher: Taylor \& Francis

Journal: Expert Review of Respiratory Medicine

DOI: $10.1080 / 17476348.2017 .1269604$

\section{REVIEW}

The use of Multiple Breath Washout for Assessing Cystic Fibrosis in Infants

\section{Authors:}

Dr Gwyneth Davies ${ }^{1,2}$ MBChB, MSc, PhD, MRCPCH.

NIHR Academic Clinical Lecturer in Paediatric Respiratory Medicine.

Dr Paul Aurora ${ }^{1,2}$ MBBS, MSc, PhD, MRCP.

Consultant and Honorary Senior Lecturer in Paediatric Respiratory Medicine.

\section{Institutions:}

${ }^{1}$ Respiratory Critical Care and Anaesthesia Section; Infection, Immunity and Inflammation Programme, UCL Great Ormond Street Institute of Child Health, London UK

${ }^{2}$ Respiratory Medicine, Great Ormond Street Hospital for Children NHS Foundation Trust, London, UK. 
Author for correspondence: gwyneth.davies@ucl.ac.uk

\begin{abstract}
Introduction: Lung Clearance Index, measured using the multiple breath washout (MBW) technique, may be a useful test in infants with Cystic Fibrosis (CF). However, the requirement for specialised equipment and a number of important technical and methodological considerations relevant to testing in infants have complicated matters, and to date prevented its widespread translatability in this age group.

Areas covered: We review the current status of infant MBW testing in CF, focusing on Lung Clearance Index. This includes a review of recent developments in the field relevant to testing methodology in the infant population, use in evaluating lung disease in $\mathrm{CF}$ in infancy, and the associated challenges which remain.
\end{abstract}

Expert commentary: The challenges of infant MBW are not limited to those associated with testing equipment, but also gaps in our understanding regarding the interpretation of MBW indices in infants. This includes their relationship to underlying physiology and pathology, and tracking over time. Recent advances in understanding and improving the infant MBW test set up (including both hardware and software) relevant to infants will greatly progress the field.

\title{
Keywords
}


Cystic fibrosis, Infant lung function, Lung Clearance Index, Multiple Breath Washout, Clinical trials. 


\section{Introduction}

Cystic fibrosis (CF) is a life shortening multi-system disease responsible for considerable morbidity, particularly affecting the respiratory system [1]. Repeated respiratory tract infections and thick respiratory secretions lead to irreversible lung damage and progressive decline in lung function, with disease progression already apparent in infancy and early childhood [2]. CF is included in national newborn screening programmes in many countries, with diagnosis made in the first few weeks of life. .

There is a timely need to establish the role of multiple breath washout (MBW) in assessing CF lung disease in the first years of life. This test has potential both in the clinical monitoring of individuals, and as an outcome measure in clinical trials. We have entered an era where drugs which target the underlying molecular defect in the cystic fibrosis transmembrane conductance regulator (CFTR) have the potential to modify the disease course [3]. Whilst great success with drugs modulating CFTR function have been achieved in a small minority of the CF population $[4,5]$, results for those with the commonest disease causing mutations have been less impressive [6] and this remains a highly active research area where optimal outcome measures for clinical trials are of key importance. Many outstanding questions remain, including whether such treatments should start in early life. It is therefore important that we have robust and clinically meaningful outcome measures in place relevant to this population.

Interest in MBW as a lung function test in CF led to a workshop being convened by the North American Cystic Fibrosis Foundation in 2014 [7], to assess its role in multi-centre clinical trials across the paediatric age range, and in routine clinical care. Lung Clearance Index (LCI), measured using the MBW technique, has been suggested as a potential suitable test in 
infants with CF. However, there are a number of important methodological considerations relevant to testing in infants have complicated matters and to date prevented the widespread translatability of this test in this age group. Whilst acknowledging the evidence base for preschool age group, the workshop report listed that "gaps in knowledge about the choice of device, gas, and standardization across systems are key issues precluding its use as a clinical trial end point in infants", and that "based on the current evidence, there are insufficient data to support the use of LCI or MBW parameters in the routine clinical management of patients with CF." [7].

This article reviews the current status of infant MBW testing in cystic fibrosis, focusing on LCI. It includes a review of recent developments in the field relevant to testing methodology in the infant population, use in evaluating lung disease in CF in infancy, and the associated challenges. Many of the methodological issues associated with testing are not specific to infants with CF, but have the potential to significantly impact on results.

\section{Multiple breath washout}

The MBW technique was first described over 60 years ago [8], as a means of assessing homogeneity of yentilation and measurement of functional residual capacity (FRC). Its potential role in $\mathrm{CF}$ has been the focus of significant research interest, particularly over the past decade. The general principles of MBW testing are essentially summarised by its title the 'washout' (i.e. the elimination) of a gas during tidal breathing is measured to determine clearance from the lungs and provide information on 'ventilation homogeneity' (efficiency of gas mixing). Higher LCI values reflect increased ventilation inhomogeneity (i.e. inefficient gas mixing). There are two main approaches - one involves 'washing out' resident nitrogen $\left(\mathrm{N}_{2}\right)$ from the lungs with $100 \%$ oxygen, and the other uses an inert foreign tracer gas which is 
first 'washed in' and then 'washed out' of the lungs. For the latter, gases including sulphur hexafluoride $\left(\mathrm{SF}_{6}\right)$, helium and argon have been used.

In $\mathrm{N}_{2}$ washouts, resident nitrogen within the lung is washed out by breathing in $100 \%$ oxygen. In tests using a tracer gas such as $\mathrm{SF}_{6}$, the subject inspires a gas mixture containing a fixed percentage of this tracer until there is equilibrium between inspired and expired gas concentrations. This completes the washin stage of the test, at which point the test gas is disconnected and washout commences with the subject switched to breathing air. Measurement of expired gas allows detection of the point at which the end tidal concentration of the tracer gas (or $\mathrm{N}_{2}$ in the case of nitrogen washouts) falls below $1 / 40^{\text {th }}$ of its initial concentration. Along with the simultaneous measurement of flow, these permit calculation of FRC by dividing the volume of exhaled tracer gas by its concentration in the lungs just before the start of the washout. Importantly, dead space created by equipment (e.g. facemask) is taken account of during calculations.

It is increasingly recognised that the results derived from a MBW test using a tracer gas such as $\mathrm{SF}_{6}$, in comparison to those using $\mathrm{N}_{2}$ and $100 \%$ oxygen, are not interchangeable. Reasons for this include the potential for hypoventilation with inspiration of $100 \%$ oxygen, the contribution of endogenous tissue nitrogen when an $\mathrm{N}_{2}$ methodology is used, and that cut-off points for terminating washouts using tracer gases have been based on the original research involying $\mathrm{N}_{2}$ washouts and these may not be interchangeable.

In observational research studies and clinical trials involving children and adults with $\mathrm{CF}$, the main outputs from MBW tests have focused on Lung Clearance Index (LCI). Other indices such as moment ratios and phase III slope analysis remain largely in the research arena. The test is performed at rest, during tidal breathing and does not involve any forced expiratory manoeuvre hence its attractiveness for testing involving infants and young children. The LCI 
represents the number of lung volume turnovers to reduce the expired test gas concentration to $1 / 40^{\text {th }}$ of the initial gas concentration $(\mathrm{LCI}=$ cumulative expired volume / functional residual capacity). It is important to appreciate that the LCI is therefore only able to assess ventilation homogeneity across regions of lung that are in communication during tidal breathing. The presence of e.g. mucus totally occluding an airway (as may occur in CF) would prevent assessment of ventilation inhomogeneity distal to that point, even if the distal airways were significantly diseased.

\section{MBW tests in infants}

MBW testing in infants involves a face mask being attached to a flowmeter and gas analyser, with putty used to ensure a good seal around the mask and prevent leak. Although MBW tests in older subjects with significant CF lung disease may be prolonged, tests in infants are generally shorter as younger children have a higher respiratory rate and milder lung disease. An $\mathrm{SF}_{6}$ washout in an infant can be completed within five minutes [9], with the entire test (i.e. 3 washout runs) completed within 20 minutes.

There are important differences between equipment set up for MBW testing between infants, pre-school children and older subjects. These include the position of the subject (infant MBW tests are carried out with the subject supine in quiet sleep, whereas in pre-school children through to adulthood are carried out whilst sitting and awake). The supine position has the potential to influence the FRC, lung mechanics, and distribution of ventilation [9], however with the exception of a small study involving 12 infants and children $\leq 3$ years which found no difference in FRC between MBW tests performed supine and semi-erect in an infant safety seat [10], the authors are not aware of any other literature regarding the influence of body position in this age group. 
At our centre, infant lung function tests including MBW are carried out with oral sedation using chloral hydrate. Although this permits controlled testing conditions it may contribute to atelectasis, and tests cannot be repeated within short time intervals. MBW can also be carried out in unsedated infants during quiet sleep, particularly during the first 4 months of life but variability between tests may be greater [11].

A further challenge for infant MBW tests is the influence of lung volumes on LCI.

Schmalisch et al recently demonstrated that LCI was reduced in infants followed up after admission to neonatal intensive care with wheeze, and reported that this reduction was due to differences in lung volumes rather than reduced ventilation inhomogeneity [12]. The authors suggested that the association between LCI and lung volumes in infants could potentially lead to difficulties in interpreting homogeneity of ventilation.

\section{MBW testing equipment and applicability to infant testing}

The original studies involving MBW tests in infants with CF were conducted using a respiratory mass spectrometer (AMIS 2000, Innovision A/S, DK) and $\mathrm{SF}_{6}$ as the tracer gas [13], and has been considered the gold standard for testing. The development of commercial devices for the measurement of LCI in older children and adults has largely focused on nitrogen washouts (e.g. Exhalyzer D (Ecomedics AG, Switzerland) and EasyOne Pro (ndd Medizintechnik AG, Switzerland), however the Exhalyzer D has an option to use a tracer gas $\left(4 \% \mathrm{SF}_{6}\right)$ for testing in infants, [14-18]. There are some concerns regarding the optimal configuration for the latter, and further modifications to the system are ongoing. Custom adaptations to the open circuit MBW method using the Innocor photoacoustic analyser (Innovision $\mathrm{A} / \mathrm{S}, \mathrm{DK}$ ) with $0.2 \% \mathrm{SF}_{6}$, discussed later in this review, have also been proposed to make it more suitable for infant testing. To the author's knowledge, neither the 
commercially approved EasyOne Pro nor the closed circuit Innocor device for MBW testing have been tested in infants. A further challenge for the infant field is that the AMIS respiratory mass spectrometer is no longer commercially available and thus limited to those centres with an existing machine.

The preference for $\mathrm{SF}_{6}$ over nitrogen washouts in infants has centred on the concerns that breathing in $100 \%$ oxygen will alter the respiratory drive and result in hypoventilation $[19$, 20], in turn affecting the variability of tidal volumes [21]. The influence of oxygen on breathing patterns in infants has been investigated by Singer et al [22]. They looked at the effect of inspiring $100 \%$ oxygen, and any potential benefit of giving a period of $40 \%$ oxygen to induce tolerance to the higher $(100 \%)$ concentration in an effort to avert any impact on breathing patterns, in healthy controls $(n=24)$ and infants with $\mathrm{CF}$ ( $\mathrm{n}=15 ; 8$ sedated) using an ultrasonic flowmeter setup. Hypoventilation occurred in both protocols, however within the two stage approach ( $40 \%$ increased to $100 \%$ oxygen), tolerance to hyperoxia occurred. The authors suggest this incremental protocol as a means of eliminating the effect on breathing patterns when nitrogen washouts using $100 \% \mathrm{O}_{2}$ are used [22], and provide an interesting alternative to $\mathrm{SF}_{6}$ based tests in infancy.

Use of $\mathrm{SF}_{6}$ is not problem free - the gas has harmful environmental impact associated with climate change[23]. Mass spectrometers and ultrasonic flowmeter set ups using $\mathrm{SF}_{6}$ typically use around 4\% tracer gas, whereas the open circuit modified Innocor uses a much lower concentration $(0.2 \%)[24]$.

It is not possible to simply combine or consider equivalent MBW tests using different equipment set ups, as the results are not comparable. Discrepancies exist due to factors such as equipment dead space, type of washout ( $\mathrm{N}_{2}$ or tracer gas), and settings within software for analysis (either custom made or commercial). Detailed standard operating procedures are 
needed to minimise differences between centres using the same equipment. The requirement for specialised equipment, appropriately trained staff and (where performed) the ability to manage sedated infants has meant that infant MBW testing remains largely in the research arena.

\section{Masks and dead space}

The volume of dead space contributed to by e.g. the face mask used for testing has particular importance in infancy given the relative large ratio of equipment dead space to an individual's lung volumes. Meticulous attention is given by those performing infant MBW tests to minimise this, as different dead space volumes (e.g. between types of mask) can have a significant impact on LCI results [25, 26]. Benseler et al [27] have recently reported the impact of dead space volume on LCI results using both a nitrogen washout set up (Exhalyzer D), and $\mathrm{SF}_{6}$ set up (using the Amis 2000 respiratory mass spectrometer, Innovision $\mathrm{A} / \mathrm{S}$ ) with in vivo measurements performed in adults (and then pre-schoolers with CF and controls), and applied their results to the impact on LCI in pre-schoolers. They found that increases in equipment dead space resulted in higher LCI when using the nitrogen MBW set up, and that differences in LCI values when measured by the two systems were reduced when the dead space was equivalent in each [27], although the contribution of endogenous tissue nitrogen and potentialimpact on breathing patterns resulting from $100 \%$ oxygen may also contribute to differences between setups.

\section{Influence of software algorithms on infant $\mathrm{MBW}$ in $\mathrm{CF}$}

The analysis of MBW tests is dependent on software specific to the set up, often conducted off line at conclusion of testing. With the increasing availability of commercially accessible equipment and software for MBW testing, the move has been towards automating output and 
minimising the input required from the operator for washout settings. Using an ultrasonic flowmeter MBW set up (Exhalyzer D) with $4 \% \mathrm{SF}_{6}$ as the tracer gas and Wbreath software (ndd, Medizintechnik AG, Zurich, Switzerland) Anagnostopoulou et al reported that the software algorithm used influenced the LCI result for infants with CF [28]. The method relies on an indirect calculation of the inert gas concentration by analysing changes in mainstream molar mass. Anagnostopoulou found that by applying automatic rather than manual settings for the software algorithms resulted in a significantly lower LCI, largely due to discrepancies between the two algorithms in detecting the end of the washout [28].

\section{MBW set ups and accuracy in infancy}

One reason for the attractiveness of mass spectrometry for infant MBW has reflected their rapid gas analyser times. A very fast response time is important in infants due to their increased respiratory rate in comparison to older subjects. In this respect, a limitation of the open circuit Innocor MBW set ups has been in its gas analyser response time exceeding 100 milliseconds. However in-house adaptations to the Innocor device have been performed by Horsley, which reduced this from 154 to 88 milliseconds [29], and tested in a lung model system for accuracy of FRC volume calculation. At lung volumes relevant to infant testing, these modifications were successful in FRC measurement with a high degree of accuracy.

In view of the challenges of reducing dead space volumes, and difficulties with accessing mass spectrometry Shawcross et al have recently described a set up for MBW tests which in in vitro testing using a simulated lung model, suggest may have a role in infant testing [30]. Their set up uses the Innocor device with $\mathrm{SF}_{6}$ as the tracer gas, but instead of integrating a flowmeter within the testing circuit, the expired gas is analysed at termination of the washout, following which the cumulative expired volume, FRC and therefore LCI are calculated. The absence of inclusion of a flowmeter within the testing circuit leads to a reduction in deadspace, and calculation of LCI does not rely on the integration of flow and gas signals. 
Shawcross et al have determined that this set up resulted in an accurate FRC measurements (within $5 \%$ in $>90 \%$ tests).

Accuracy of the ultrasonic flowmeter Exhalzyer D (EcoMedics) has also been investigated using this lung model. Singer et al investigated the Exhalyzer D nitrogen washout and reported that $43 \%$ tests met this same degree of accuracy in their "small lung" model (100400ml FRC), with more favourable results in tests with higher FRC [31]. More recently, using the same lung model as Singer, Schmidt et al investigated two ExhalyzerD MBW set ups, using $\mathrm{SF}_{6}$ and helium as tracer gases [32]. They used $\mathrm{SF}_{6}(4 \%)$ for the infant set up and helium (20\%) for a 'young child' set up. In their infant lung model, the MBW SF 6 set up gave a relative difference between model and measured FRC of mean(SD) $0.7(4.7) \%$ at volumes between $80-300 \mathrm{ml}$.

\section{Improving the feasibility of $\mathrm{MBW}$ in infants}

The ERS/ATS consensus recommendations for MBW testing recommend that mean FRC is reported from three technically acceptable measurements [33]. Pre-school guidelines for MBW had recommended that two technically acceptable tests with FRC within $10 \%$ of each other were sufficient for calculating mean FRC and LCI [34]. Robinson et al investigated the feasibility of using this 'abbreviated' MBW testing protocol across the paediatric age range, from infancy, in healthy controls and $\mathrm{CF}$ [35]. They concluded that the recommendation for two rather than three technically acceptable tests (irrespective of differences in FRC between tests) was appropriate and that this did not unduly affect LCI.

Although not from infants, Yammine et al used nitrogen MBW (using the Exhalyzer D, EcoMedics AG, Switzerland) in school age children to look at the influence on test sensitivity and specificity and duration, using a number of strategies aimed at shortening the test (and thereby improving its acceptability). They found that performing either three MBW runs to 
$1 / 20^{\text {th }}$ (rather than the 'standard' $1 / 40^{\text {th }}$ ) of the nitrogen starting concentration, or two runs until $1 / 40^{\text {th }}$ both significantly reduced the time taken for MBW testing in children without adversely affecting diagnostic performance [36].

Respiratory sighs in infants have been suggested as exclusion criteria for analysis in MBW testing recommendations [33]. MBW was measured in infants between 4-12 weeks of age during natural sleep using an ultrasonic flowmeter and $\mathrm{SF}_{6}$ as the tracer gas (Exhalyzer $\mathrm{D}$, Ecomedics) by Vukcevic et al. They found that sighs occurred in 119/767 (15\%) of infant MBW tests and that when occurring in the washout phase of testing impacted on results, with sighs resulting in elevated LCI (mean $(95 \% \mathrm{CI})$ LCI increased by $0.36(0.11$ to 0.62$)$ units with variance increasing by a factor of two) [37]. However, there was negligible impact on LCI when sighs occurred during the washin phase, leading the authors to suggest that these tests need not be excluded thus increasing the proportion of technically satisfactory tests.

\section{MBW indices other than LCI}

As an outcome from the MBW test, LCI has received by far the most attention but many indices can be calculated and some are being subjected to renewed interest [33]. These indices are often accompanied by their own challenges in terms of relevance and interpretation for infant MBW. For example, moment ratios (which take into account area under the washout curve) are reported far less commonly than LCI, and methodology appropriate to infant tests has not been standardised. More generally, it may be that historically used cut points to indicate termination of the MBW test need some adjustment to optimise the discriminatory ability of LCI to detect ventilation inhomogeneity. Furthermore, the assumption that the same cut of $\left(1 / 40^{\text {th }}\right)$ is equitable between washouts using different tracer gases has recently been challenged. Yammine et al compared the impact of using a $2.5 \%\left(1 / 40^{\text {th }}\right)$ cut off for terminating a nitrogen and foreign (e.g. $\left.\mathrm{SF}_{6}\right)$ tracer gas washout (by 
taking account of endogenous nitrogen to make the nitrogen washout comparable to one carried out using a foreign tracer gas)[38]. They found that the contribution of tissue nitrogen had a sizable, clinically relevant impact on LCI and FRC and suggested that a cut off of $1.5 \%$ for $\mathrm{SF}_{6}$ washouts may be more comparable to $\mathrm{N} 2$ washouts using $2.5 \%$ cut off.

Egger et al have investigated the use of the $1 / 40^{\text {th }}$ cut point (i.e. $2.5 \%$ of the starting tracer gas concentration) in $\mathrm{SF}_{6}$ washouts for both LCI and moment ratios in infancy. They found optimal discriminative power for moment ratios and LCI to detect differences between healthy controls and infants with cystic fibrosis at 1.5\% tracer gas concentration [39].

\section{The role of reference data for interpreting infant $\mathrm{LCI}$}

In older children ( $>6$ yrs) and adults, LCI is stable in health and therefore can be reported as an absolute value. In the early years, LCI has been noted to be elevated and therefore standardization with z scores permits comparison between longitudinal measurements. Collated data from three centres was used to investigate the age and height dependence of lung clearance index in health. Lum et al collected data on nearly 500 children across the paediatric age range, from 2 weeks to 19 years of age, on $>650$ occasions [40]. Mass spectrometry with $4 \% \mathrm{SF}_{6}$ was used for the MBW tests. They found that there was an age and height dependence of LCI in infancy, with LCI dependent on body size. Particularly in the first five years of life, LCI was found to decrease non-linearly with increasing height. Height, age and sex were all independent predictors of FRC. Reference equations for the calculation

of z scores using the lambda-mu-sigma (LMS) method were created, which permit interpretation of results. However it is important to note that these equations will only be applicable to LCI as measured with the same test set-up, creating an added complexity to a field where comparisons between centres and devices is challenging. Conversion of LCI to z scores using data from healthy controls obtained using the same equipment is important for 
interpretation particularly when tracking disease over time. This in itself creates a challenge for the field. It is important that reference equations are relevant to the equipment being used and the population of interest, and that if appropriate reference data is not available, measurements are made on contemporaneous healthy controls in order to benchmark the results appropriately.

\section{Relationship to other infant lung function tests}

The order in which infant lung function tests are performed has the potential to influences the results obtained, as such careful consideration must be given when planning testing protocols which involve multiple lung function tests in addition to MBW. Subbarao and colleagues have looked at the influence of raised volume rapid thoracic compression (RVRTC) manoeuvres on MBW measurements in infants [41]. Their testing protocol sequence involved MBW, RVRTC, and then repeat MBW, with infants sedated using oral chloral hydrate and using the AMIS mass spectrometer (Innovision, DK) with an $\mathrm{SF}_{6} /$ Helium gas mixture. They found that performing the forced respiratory manoeuvres in RVRTC testing prior to MBW resulted in reductions in FRC and (to a greater extent) cumulative expired volume, with such changes particularly noted in infants with respiratory disease (CF or wheeze).

\section{2. $\mathrm{MBW}$ in infants with $\mathrm{CF}$}

To date, it has not been clearly established how infant LCI measurements relate to those performed in later childhood. It is also important that we determine the implications of LCI values in infancy, and how they relate to structural abnormalities, clinical parameters, and infection and inflammation on a cross sectional and longitudinal basis. It should also be remembered that $\mathrm{SF}_{6}$ and $\mathrm{N}_{2}$ washouts are not interchangeable, yet the bulk of evidence which has been built up over the past two decades on the utility of MBW and LCI in CF lung disease was carried out using the $\mathrm{SF}_{6}$ method and respiratory mass spectrometry. Therefore 
we cannot be certain that results from $\mathrm{N}_{2}$ washouts would correlate with other parameters or represent the extent of disease in the same way. 12.1 Change in LCI over time

In infants with CF diagnosed by newborn screening (NBS), the proportion with an abnormal LCI at around 3 months of age and 1 year is relatively low. The London Cystic Fibrosis Collaboration (LCFC, https://www.ucl.ac.uk/london-cystic-fibrosis) is following up a cohort of NBS infants with CF, and contemporaneous healthy controls. CF infants and healthy controls had lung function tests performed under sedation at around 3 months of age and one year, including MBW with respiratory mass spectrometry (AMIS 2000) and SF$_{6}$ as the tracer gas. The proportion with an abnormal LCI $(>1.96 \mathrm{z})$ at these time points was $17 \%(12 / 71)$ and $18 \%(13 / 71)$ respectively [42]. This cohort continues to be followed up, but the proportion of children with an elevated LCI in a clinically diagnosed cohort (recruited before the start of national NBS in the UK) increased during childhood [43, 44]. The Australian Respiratory Early Surveillance Team for CF (AREST-CF) have similarly reported that the vast majority of infants with CF diagnosed by NBS have normal LCI results [45], although direct comparisons with the LCFC cohort are not possible due to the use of different MBW set ups (Exhalyzer D vs AMIS 2000 mass spectrometry) and absence of contemporaneous healthy controls in the AREST-CF study.

In pre-school children with CF diagnosed clinically, LCI has been shown to track to school age [43] but an evidence base to support tracking through infant measurements is currently lacking. The LCFC has shown that LCI measured by mass spectrometry tracked from 3months to 1year of age in NBS CF infants [42], but interestingly there was no tracking between tests performed (in a non-interventional setting) at around 1 and 2 years of age [46] . Given this lack of tracking between 1-2 years of age, the clinical interpretation of an apparent 'improvement' in LCI, from those with an 'abnormal' result at early infant timepoints (e.g. 
$3 \mathrm{mths}$ ) which 'normalises' by $1 \mathrm{yr}$ or $2 \mathrm{yrs}$ remains to be determined. The LCFC has recently completed testing the NBS CF cohort at pre-school age, and results are eagerly awaited.

Although small in terms of patient numbers, a single centre retrospective study from Switzerland using the Exhalyzer D MBW set up reported paired infant and school age measurements from 11 children with $\mathrm{CF}$ (MBW measured with $\mathrm{SF}_{6}$ in infants and $\mathrm{N}_{2}$ in school age children), providing some evidence for the ability of infant LCI to track through to school age [47].

Longitudinal cohort studies such as those performed by the LCFC and AREST-CF groups will help us to understand the variability of LCI in CF, and how it changes in response to routine or novel therapies in the first years of life.

\subsection{Relation to chest CT}

Lung clearance index has been shown to correlate more closely than spirometry in pre-school and school age children with CF to structural lung disease on chest computed tomography (CT)[48-50]. In sixty school age children with CF diagnosed clinically (mean age 7.8yrs), total CT score correlated strongly with LCI, however in individual patients LCI and chest CT were considered complimentary to each other: $5 / 9$ children with normal LCI had abnormalities on HRCT, whereas five children with normal HRCT had raised LCI [50]. In infants, the relationship between LCI and structural evidence of CF lung disease on chest CT is less clear. The AREST-CF group found no association between LCI (measured using an ultrasonic flowmeter (EcoMedics, Switzerland) and 5\% $\mathrm{SF}_{6}$ ) and neither the presence of bronchiectasis nor air trapping, but that moment ratios may reflect evidence of structural lung disease in infants with CF [51]. Ramsey et al have recently investigated relationships between structural outcomes (chest CT) and LCI across the paediatric age range [45]. They looked at the association between structural lung disease and LCI measured using the Exhalyzer D 
using 4\% SF6, and in infants found poor correlation between LCI and structural lung disease $(\mathrm{k}=20.03$ [95\% confidence interval, 20.05 to 0.16$])$, with LCI having low negative predictive value for bronchiectasis and air trapping. In contrast, in older children LCI (measured with the Exhalyzer $\mathrm{D} \mathrm{N}_{2}$ set up) values were more closely associated with evidence of lung disease on chest CT: in pre-school and school age children LCI correlated with total structural disease extent and additionally at school age with extent of bronchiectasis and air trapping. An added complexity when investigating relationships between abnormal functional and structural outcomes is introduced by their prevalence - particularly if abnormalities are rare and mild, as we have observed with chest CT in NBS CF infants in the LCFC cohort [52]. The difficulties with mapping LCI to structural abnormalities do not preclude its potential usefulness in this age group, but further evidence is needed regarding the significance of an elevated LCI in the context of a normal chest CT. It may be that an elevated LCI in the absence of structural abnormalities on chest $\mathrm{CT}$ is instead reflects inflammation and infection, as described in section 12.3 below.

\subsection{Relation to infection}

The AREST-CF group recently published the association between infant LCI and pulmonary infection. They performed MBW testing and bronchoalveolar lavage(BAL) at three time points in the first 2 years of life in 108 infants with CF. They found that significant pulmonary infection (particularly with Haemophilus influenzae) was associated with concurrent elevation in LCI, and that deficits in ventilation homogeneity persisted over time [53]. Using the Exhalyzer D ultrasonic flowmeter with $\mathrm{SF}_{6}$ as the tracer gas, Belessis et al found that LCI in CF infants (mean age 1.55 years) was elevated in comparison to healthy controls, and that within the CF subjects the presence of Pseudomonas aeruginosa and evidence of airway inflammation on bronchoalveolar lavage was associated with elevated LCI [16]. 


\section{Infant LCI as an outcome measure in CF clinical trials}

LCI has established a role in the assessment of pre-school children with CF and in older subjects with normal spirometry, and has been used as an outcome measure in clinical trials. It has been used successfully as a primary outcome measure in a phase II clinical trial of the CFTR modulator ivacaftor involving CF patients aged 6yrs and over with normal spirometry (forced expiratory volume in 1 second $>90 \%$ predicted) [54], where a significant treatment effect was detected. In school age children with CF LCI has successfully demonstrated a significant treatment effect for dornase alpha and hypertonic saline $[55,56]$.

There has been limited use of MBW in clinical trials involving infants with CF to date. A clinical trial of inhaled hypertonic saline in infants and children less than 6yrs old with $\mathrm{CF}$ (the ISIS study)[57], has explored LCI as an endpoint, where it was included in the trial protocol as an exploratory outcome. This study was a multicentre, randomized, controlled trial of $7 \%$ vs $0.9 \%$ nebulised saline twice daily for 48 weeks. LCI was measured at a single site, in a sub-group of the study population. Acceptable LCI measurements were obtained in 25/27 participants at baseline and at the end of the trial, using mass spectrometry (AMIS 2000; Innovision) and $\mathrm{SF}_{6}$ as the tracer gas [57]. The median age of participants in this substudy was 2.6 years (range 0.34 to $4.95 \mathrm{yrs}$ ), crossing into the pre-school years. Infant lung function tests were performed under sedation in those $<16$ months of age in a supine position, whereas those $>16$ months were performed awake and seated. LCI results were converted to Z scores for interpretation [40]. 4/12 children in the hypertonic saline group were infants, in comparison to $6 / 13$ in the isotonic saline group. Due to the low n numbers, interpretation of

LCI results is limited. However although not powered, the authors noted that infants randomised to treatment with hypertonic saline did not show change in LCI, whereas an increased LCI (suggestive of deterioration) over the 48 week study period was observed in those receiving isotonic saline [57]. These results contrasted to those in children from the pre- 
school age group, where changes suggestive of improvement were observed in those receiving the hypertonic solution. Importantly, the authors of the ISIS study suggested that MBW testing was feasible in a single centre interventional study in infants and preschool children with CF. A challenge for all potential efficacy outcome measures in early life is how they compare to healthy controls, i.e. whether there are any detectable deficits by timepoints potentially measured by infant lung function tests such as MBW. As described above, the LCFC and AREST-CF cohort studies have both shown that the vast majority of infants with CF diagnosed by NBS have normal LCI values. In the ISIS study, all infants had a normal LCI at their baseline measurement.

\section{Challenges, conclusions and the future}

This article has reviewed the use of MBW testing in infants with $\mathrm{CF}$, along with a number of recent developments relevant to the field relating to methodology both within and between different MBW set ups. However it is not only technical aspects that need to be resolved, but also that we better understand the interpretation of MBW indices - in terms of relationship to underlying physiology and pathology - in our youngest patients with CF.

\section{Expert commentary}

The challenges of infant MBW are not limited to those associated with testing equipment, but also gaps in our understanding regarding the interpretation of MBW indices in infants. This includes their relationship to underlying physiology and pathology, and tracking over time.

Recent advances in understanding and improving the infant MBW test set up (including both hardware and software) relevant to infants will greatly progress the field. New therapies targeting the basic defect in CF make it even more important that the field embraces these outstanding questions to determine whether infant MBW should or could play a role in evaluating their efficacy, or in its role in longitudinal follow up in routine care. There remains 
a great deal of work to standardise measurements and analysis and improve the translatability of infant MBW testing. Given the rapidly evolving field of new treatments for CF which have the potential to modify disease, this is an opportunity that cannot afford to be missed.

\section{Five year view}

Over the past decade, MBW testing has established itself as a key player in evaluating CF lung disease in older children and adults with mild lung disease. However, it is likely that infant MBW testing will remain in the research setting for the foreseeable future. By five years' time we should be better placed to understand the clinical significance of infant MBW tests, and to determine which (if any) 'key' testing points in infancy track to measures made in the pre-school years and beyond.

\section{Key issues}

- Many indices can be calculated from the Multiple Breath Washout test, but the focus has been on Lung Clearance Index (LCI)

- In children of pre-school age and above, there is evidence that LCI correlates with structural lung disease on chest imaging however this relationship is less clear in infants.

- The preference for inert tracer gases such as $\mathrm{SF}_{6}$ in infant MBW stems from evidence that the high concentrations of oxygen required for nitrogen washouts affect the breathing pattern in infants and thus influence LCI.

Infant MBW testing is largely limited to the research setting. There is a single commercially available option for performing tests, and the historically considered 'gold standard' method using respiratory mass spectrometry is limited to existing centres as no longer commercially available. 
- LCI results are not consistent across different test set ups, and small changes e.g. to mask size or software settings can impact greatly on results.

- There is a need for respiratory outcome measures for clinical trials of novel treatments in the first years of life, but the evidence base to support infant MBW in this role is currently lacking.

\section{Funding:}

G. Davies is supported by a National Institute for Health Research (NIHR) Academic Clinical Lectureship at University College London, and along with PA is supported by the NIHR Biomedical Research Centre at Great Ormond Street Hospital for Children NHS Foundation Trust and University College London.

\section{Declaration of Interest}

P. Aurora is a consultant for Vertex Pharmaceuticals in relation to a future trial in infants with CF using LCI as an endpoint. He has no other affiliations or financial involvement with any organization or entity with a financial interest in or financial conflict with the subject matter or materials discussed in the manuscript. The authors have no other relevant affiliations or financial involvement with any organization or entity with a financial interest in or financial conflict with the subject matter or materials discussed in the manuscript apart from those disclosed.This article presents independent research funded by the National Institute for Health Research (NIHR). The views expressed are those of the author(s) and not necessarily those of the NHS, the NIHR or the Department of Health. 


\section{References}

Papers of special note have been annotated as:

* Of interest

** Of considerable interest

1. $\quad$ Elborn JS. Cystic fibrosis. Lancet. 2016;388(10059):2519-31.

2. VanDevanter DR, Kahle JS, O'Sullivan AK, et al. Cystic fibrosis in young children: A review of disease manifestation, progression, and response to early treatment. J Cyst Fibros. 2016;15(2):147-57.

3. Sawicki GS, McKone EF, Pasta DJ, et al. Sustained Benefit from ivacaftor demonstrated by combining clinical trial and cystic fibrosis patient registry data. Am J Respir Crit Care Med. 2015;192(7):836-42.

4. Davies JC, Wainwright CE, Canny GJ, et al. Efficacy and safety of ivacaftor in patients aged 6 to 11 years with cystic fibrosis with a G551D mutation. Am J Respir Crit Care Med. 2013;187(11):1219-25.

5. Ramsey BW, Davies J, McElvaney NG, et al. A CFTR potentiator in patients with cystic fibrosis and the G551D mutation. N Engl J Med. 2011;365(18):1663-72.

6. Wainwright CE, Elborn JS, Ramsey BW, et al. Lumacaftor-Ivacaftor in Patients with Cystic Fibrosis Homozygous for Phe508del CFTR. N Engl J Med. 2015;373(3):220-31.

7. Subbarao P, Milla, C, Aurora, P, et al. Multiple-Breath Washout as a Lung Function Test in Cystic Fibrosis. A Cystic Fibrosis Foundation Workshop Report. Ann Am Thorac Soc. 2015;12(6):932-9.

**Cystic Fibrosis Foundation Report on the current status of MBW testing in CF, including recommendations for infants

8. Becklake MR. A New Index of the Intrapulmonary Mixture of Inspired Air. Thorax. 1952;7(1):111-6. 
9. Stocks J, Lum S. 11 - Pulmonary Function Tests in Infants and Preschool Children A2

- Wilmott, Robert W. In: Boat TF, Bush A, Chernick V, Deterding RR, Ratjen F, editors. Kendig \& Chernick's Disorders of the Respiratory Tract in Children (Eighth Edition). Philadelphia: W.B. Saunders; 2012. p. 169-210.

10. Aljassim F, Lindblad A, Gustafsson P. Effects of Body Posture on FRC and LCI in CF Children. Am J Respir Crit Care Med. 2009;179(abstract):A1776.

11. Sinhal S, Galati J, Baldwin DN, et al. Reproducibility of multiple breath washout indices in the unsedated preterm neonate. Pediatr Pulmonol. 2010;45(1):62-70.

12. Schmalisch G, Wilitzki S, Buhrer C, et al. The lung clearance index in young infants: impact of tidal volume and dead space. Physiol Meas. 2015;36(7):1601-13.

13. Aurora P, Kozlowska W, Stocks J. Gas mixing efficiency from birth to adulthood measured by multiple-breath washout. Respir Physiol Neurobiol. 2005;148(1-2):125-39.

14. Schibler A, Hall GL, Businger F, et al. Measurement of lung volume and ventilation distribution with an ultrasonic flow meter in healthy infants. Eur Respir J. 2002;20(4):912-8. 15. Hulskamp G, Lum S, Stocks J, et al. Association of prematurity, lung disease and body size with lung volume and ventilation inhomogeneity in unsedated neonates: a multicentre study. Thorax. 2009;64(3):240-5.

16. Belessis Y, Dixon B, Hawkins G, et al. Early cystic fibrosis lung disease detected by bronchoalveolar lavage and lung clearance index. Am J Respir Crit Care Med. $2012 ; 185(8): 862-73$.

17. Fuchs O, Latzin P, Thamrin C, et al. Normative data for lung function and exhaled nitric oxide in unsedated healthy infants. Eur Respir J. 2011;37(5):1208-16.

18. Stahl M, Joachim C, Blessing K, et al. Multiple Breath Washout Is Feasible in the Clinical Setting and Detects Abnormal Lung Function in Infants and Young Children with Cystic Fibrosis. Respiration. 2014;87(5):357-63. 
19. Brady JP, Cotton EC, Tooley WH. Chemoreflexes in the newborn infant: Effects of 100 per cent oxygen on heart rate and ventilation.. J Physiol. 1964;172:332-41.

20. Mortola JP, Frappell PB, Dotta A, et al. Ventilatory and metabolic responses to acute hyperoxia in newborns. Am Rev Respir Dis. 1992;146(1):11-5.

21. Schibler A, Schneider M, Frey U, et al. Moment ratio analysis of multiple breath nitrogen washout in infants with lung disease. Eur Respir J. 2000;15(6):1094-101.

22. Singer F, Yammine S, Schmidt A, et al. Ventilatory response to nitrogen multiplebreath washout in infants. Pediatr Pulmonol. 2014;49(4):342-7.

23. Dervos CT, Vassiliou P. Sulfur hexafluoride $\left(\mathrm{SF}_{6}\right)$ : globalenvironmental effects and toxic byproduct formation. J Air Waste Manag Assoc. 2000;50(1):137-41.

24. Horsley AR, Gustafsson PM, Macleod KA, et al. Lung clearance index is a sensitive, repeatable and practical measure of airways disease in adults with cystic fibrosis. Thorax. 2008;63(2):135-40.

25. Schmalisch G, Proquitte H, Roehr CC, et al. The effect of changing ventilator settings on indices of ventilation inhomogeneity in small ventilated lungs. BMC Pulm Med. 2006;6:20.

26. Schulzke SM, Hall GL, Nathan EA, et al. Lung Volume and Ventilation Inhomogeneity in Preterm Infants at 15-18 Months Corrected Age. Journal of Pediatrics. 2010;156(4):542-U61.

27. Benseler A, Stanojevic S, Jensen R, et al. Effect of equipment dead space on multiple breath washout measures. Respirology. 2015;20(3):459-66.

28. Anagnostopoulou P, Yammine S, Schmidt A, et al. False Normal Lung Clearance Index in Infants With Cystic Fibrosis Due to Software Algorithms. Pediatr Pulmonol. 2015;50(10):970-7.

*Study demonstrating the impact of software settings on infant MBW results 
29. Horsley A, Macleod K, Gupta R, et al. Enhanced Photoacoustic Gas Analyser Response Time and Impact on Accuracy at Fast Ventilation Rates during Multiple Breath Washout. Plos One. 2014;9(6):e98487.

30. Shawcross A, Murray CS, Goddard N, et al. Accurate lung volume measurements in vitro using a novel inert gas washout method suitable for infants. Pediatr Pulmonol. 2016;51(5):491-7.

31. Singer F, Houltz B, Latzin P, et al. A realistic validation study of a new nitrogen multiple-breath washout system. PLoS One. 2012;7(4):e36083.

32. Schmidt A, Yammine S, Proietti E, et al. Validation of multiple-breath washout equipment for infants and young children. Pediatr Pulmonol. 2015;50(6):607-14.

33. Robinson PD, Latzin P, Verbanck S, et al. Consensus statement for inert gas washout measurement using multiple- and single- breath tests. Eur Respir J. 2013;41(3):507-22.

34. Beydon N, Davis SD, Lombardi E, et al. An official American Thoracic Society/European Respiratory Society statement: pulmonary function testing in preschool children. Am J Respir Crit Care Med. 2007;175(12):1304-45.

35. Robinson PD, Stocks J, Aurora P, et al. Abbreviated multi-breath washout for calculation of lung clearance index. Pediatr Pulmonol. 2013;48(4):336-43.

36. Yammine S, Singer F, Abbas C, et al. Multiple-breath washout measurements can be significantly shortened in children. Thorax. 2013;68(6):586-7.

37. Vukcevic D, Carlin, JB, King, L, et al. The influence of sighing respirations on infant lung function measured using multiple breath washout gas mixing techniques. Physiol Rep. 2015;3(4).(pii):e12347.

38. Yammine S, Lenherr N, Nyilas S, et al. Using the same cut-off for sulfur hexafluoride and nitrogen multiple-breath washout may not be appropriate. J Appl Physiol (1985). 2015;119(12):1510-2. 
39. Egger B, Jost, K., Anagnostopoulou, P, et al. Lung clearance index and moment ratios at different cut-off values in infant multiple-breath washout measurements. Pediatr Pulmonol. 2016;23(10):23483

40. Lum S, Stocks J, Stanojevic S, et al. Age and height dependence of lung clearance index and functional residual capacity. Eur Respir J. 2013;41(6):1371-7.

*LCI measured using a respiratory mass spectrometry and $\mathrm{SF}_{6}$ is dependent on age and height in the first years of life

41. Subbarao P, Lu Z, Kowalik K, et al. Changes in multiple breath washout measures after raised volume rapid thoracoabdominal compression maneuvers in infants. Pediatr Pulmonol. 2016;51(2):183-8.

42. Nguyen TT, Thia LP, Hoo AF, et al. Evolution of lung function during the first year of life in newborn screened cystic fibrosis infants. Thorax. 2014;69(10):910-7.

\section{** Longitudinal changes in infant lung function in CF between $3 \mathrm{mths}$ and $1 \mathrm{yr}$ of age}

43. Aurora P, Stanojevic S, Wade A, et al. Lung clearance index at 4 years predicts subsequent lung function in children with cystic fibrosis. Am J Respir Crit Care Med. 2011;183(6):752-8.

44. Aurora P, Gustafsson P, Bush A, et al. Multiple breath inert gas washout as a measure of ventilation distribution in children with cystic fibrosis. Thorax. 2004;59(12):1068-73.

45. Ramsey KA, Rosenow T, Turkovic L, et al. Lung Clearance Index and Structural Lung Disease on Computed Tomography in Early Cystic Fibrosis. Am J Respir Crit Care Med. 2016;193(1):60-7.

*The associations between LCI and structural lung disease are unclear in infants with CF.

46. Davies G, Thia L, Hoo A-F, et al. Within-subject variability of lung function in newborn screened (NBS) CF infants (abstract). Eur Respir J. 2016;48(suppl 60). 
47. Kieninger E, Singer F, Fuchs O, et al. Long-term course of lung clearance index between infancy and school-age in cystic fibrosis subjects. J Cyst Fibros. 2011;10(6):487-90. 48. Gustafsson PM, De Jong PA, Tiddens HA, et al. Multiple-breath inert gas washout and spirometry versus structural lung disease in cystic fibrosis. Thorax. 2008;63(2):129-34. 49. Ellemunter H, Fuchs SI, Unsinn KM, et al. Sensitivity of Lung Clearance Index and chest computed tomography in early CF lung disease. Respir Med. 2010;104(12):1834-42. 50. Owens CM, Aurora P, Stanojevic S, et al. Lung Clearance Index and HRCT are complementary markers of lung abnormalities in young children with CF. Thorax. $2011 ; 66(6): 481-8$.

51. Hall GL, Logie KM, Parsons F, et al. Air trapping on chest CT is associated with worse ventilation distribution in infants with cystic fibrosis diagnosed following newborn screening. PLoS One. 2011;6(8):e23932.

52. Thia LP, Calder A, Stocks J, et al. Is chest CT useful in newborn screened infants with cystic fibrosis at 1 year of age? Thorax. 2014;69(4):320-7.

53. Simpson SJ, Ranganathan S, Park J, et al. Progressive ventilation inhomogeneity in infants with cystic fibrosis after pulmonary infection. Eur Respir J. 2015;46(6):1680-90.

54. Davies J, Sheridan H, Bell N, et al. Assessment of clinical response to ivacaftor with lung clearance index in cystic fibrosis patients with a G551D-CFTR mutation and preserved spirometry: a randomised controlled trial. Lancet Respir Med. 2013;1(8):630-8.

\section{*LCI as primary outcome measure in clinical trial involving children age 6 and over}

\section{with normal spriometry}

55. Amin R, Subbarao P, Lou W, et al. The effect of dornase alfa on ventilation inhomogeneity in patients with cystic fibrosis. Eur Respir J. 2011;37(4):806-12.

56. Amin R, Subbarao P, Jabar A, et al. Hypertonic saline improves the LCI in paediatric patients with CF with normal lung function. Thorax. 2010;65(5):379-83. 
57. Subbarao P, Stanojevic S, Brown M, et al. Lung Clearance Index as an Outcome Measure for Clinical Trials in Young Children with Cystic Fibrosis A Pilot Study Using Inhaled Hypertonic Saline. Am J Respir Crit Care Med. 2013;188(4):456-60.

** LCI as an exploratory end point in a clinical trial involving infants and pre-school children with CF. 